Como citar este artigo: BONETTI, DÉBORA F; DE SOUZA, LUCAS; RESMINI, MARCUS B.; Avaliação do perfil epidemiológico de pacientes atendidos no ambulatório de reumatologia das clínicas integradas de uma universidade. Revista Saúde (Sta. Maria). 2020; $46(1)$.

Autor correspondente:

Nome: Débora Fornasa Bonetti E-mail: deborafbonetti@gmail.com Telefone: (48) 999779783 Formação Profissional: Graduanda em Medicina pela Universidade do Extremo Sul Catarinense (UNESC) que fica na cidade de Criciúma, Santa Catarina, SC, Brasil.

Filiação Institucional: Universidade do Extremo Sul Catarinense - UNESC

Endereço para correspondência: Rua: 26 de Maio n’: 55

Bairro: Bairro da Figueira

Cidade: Urussanga

Estado: Santa Catarina

CEP: 88840-000

Data de Submissão:

12/05/2019

Data de aceite:

16/01/2020

Conflito de Interesse: Não há conflito de interesse

\section{Avaliação do perfil epidemiológico de pacientes atendidos no ambulatório de reumatologia das clínicas integradas de uma universidade}

\section{Evaluation of the epidemiological profile of patients attended in the reumatology ambulatory of integrated clinics of a university}

Debora Fornasa Bonetti, Lucas De Souza, Marcus Barg Resmini

\section{RESUMO}

Objetivo: Avaliar o perfil epidemiológico de pacientes atendidos no serviço de reumatologia de uma universidade do extremo sul catarinense. Métodos: foi realizado um estudo de prontuários das primeiras consultas de pacientes atendidos no ambulatório de reumatologia das clínicas integradas de uma Universidade do Extremo Sul Catarinense no período de julho de 2016 até julho 2018. Resultados: A amostra estudada se constituiu de pacientes femininos, na sua maior prevalência, com idade média de 55 anos, que agendou livremente sua consulta, devido queixa de dor musculoesquelética crônica. Os principais diagnósticos foram osteoartrite, e artrite reumatoide dentre as autoimunes. Conclusões: Concluiu-se que o perfil dos pacientes atendidos nesse serviço médico é compatível com o descrito na literatura. Ressalta-se a importância do estudo por fornecer informações úteis para formulação de estratégias que visem aperfeiçoar os serviços prestados e melhor aproveitamento de seus recursos.

PALAVRAS-CHAVE: Epidemiologia; Reumatologia; Doença Crônica.

\section{ABSTRACT}

Objective: Evaluating the epidemiological profile of patients treated at the rheumatology service of a university in the extreme south of Santa Catarina. Methods: A study of medical records of the first consultations of patients seen at the rheumatology outpatient clinic of the integrated clinics of a University of Southern Santa Catarina from July 2016 to July 2018 was performed. Results: The study sample consisted of female patients in the its highest prevalence, with a mean age of 55 years, which freely scheduled their appointment, due to complaints of chronic musculoskeletal pain. The main diagnoses were osteoarthritis, and rheumatoid arthritis among the autoimmune. Conclusions: It was concluded that the profile of patients treated at this medical service is compatible with that described in the literature. We emphasize the importance of the study because it provides useful information for the formulation of strategies aimed at improving the services provided and better use of their resources.

KEYWORDS: Epidemiology; Rheumatology; Chronic Diseases. 


\section{INTRODUÇÃO}

A reumatologia é caracterizada por uma grande variedade de doenças, não apenas reumáticas sistêmicas e inflamatórias, mas também doenças degenerativas articulares e da coluna, reumatismos de partes moles e doenças ósseas metabólicas. Estas patologias representam a segunda maior causa de incapacidade e o quarto maior impacto na saúde geral da população mundial em termos de morte e incapacidade ${ }^{1,2}$.

É importante que se conheça o perfil de pacientes que utilizam serviços médicos especializados, como um ambulatório de reumatologia, visando definir se estes serviços são utilizados de forma apropriada, pela população certa e em tempo hábil, assim como otimizar os serviços prestados ${ }^{3}$.

O conhecimento sobre a doença e as características dos pacientes atendidos em ambulatórios especializados pode fornecer informações relevantes para melhorar a disponibilidade destes serviços. A otimização da eficiência de um serviço geralmente depende da promoção da consciência geral sobre o seu modo de funcionamento, o diagnóstico e 0 tratamento esclarecidos e da minimização dos atrasos na obtenção de consultas. Os atrasos no processo de atendimento são causadores de ansiedade entre os pacientes e geram, por consequência, aumento da morbidade 4 . Em nosso meio, dados apontam que a espera por uma consulta reumatológica em centros especializados pode demorar em média 7 a 8 meses $^{5}$, podendo chegar a 12 meses, dependendo da localização avaliada ${ }^{6}$.

O delineamento do perfil epidemiológico da população candidata ao atendimento de consultas em ambulatórios especializados constitui uma ferramenta de grande utilidade para planejamento administrativo e melhor funcionamento local $^{3}$. Este perfil atua como fonte identificadora de informações sobre fatores de risco para determinadas condições médicas, e também possui um papel crucial como relator de problemas no diagnóstico e seguimento do tratamento clínico ${ }^{7}$. Uma melhora na gestão do atendimento, desde a porta de entrada até o desfecho, interage efetivamente e pode melhorar os resultados finais ${ }^{8}$.

O modo e motivo de encaminhamento também têm implicações significativas para pacientes, profissionais do sistema de saúde e custos de assistência médica. Encaminhamentos apropriados para reumatologistas podem levar a melhores desfechos clínicos, bem como custos reduzidos através do uso ideal de serviços médicos, hospitalares e laboratoriais ${ }^{9}$. Adicionalmente, encaminhamentos excessivos ou mal direcionados podem levar a cuidados fragmentados (como testes repetitivos e uso inadequado de especialistas) e reduzir a disponibilidade de serviços para pacientes que realmente necessitam de acesso imediato e cuidados de longo prazo. Não há um consenso para definir o que constitui um encaminhamento reumatológico apropriado ${ }^{10}$.

Dor é certamente a razão mais comum pela qual pessoas procuram atendimento médico, sendo um sintoma particularmente importante na reumatologia ${ }^{11}$. Por este sintoma ser inespecífico observa-se elevada taxa de pacientes com queixas álgicas diversas utilizando serviços especializados de reumatologia de forma inapropriada, com uma grande parcela destes pacientes passíveis de serem atendidos e manejados em um ambiente de atenção primária. Programas 
de triagem de encaminhamentos parecem ser efetivos para a melhora deste aspecto ${ }^{12}$.

\section{OBJETIVO}

O objetivo principal desta pesquisa foi avaliar as características demográficas, clínicas e motivos de consulta de pacientes novos atendidos no ambulatório de reumatologia do serviço de medicina de uma universidade do extremo sul catarinense, no período de julho de 2016 a julho de 2018.

\section{MÉTODO}

Realizou-se um estudo retrospectivo, descritivo, com coleta de dados secundários e de natureza quantitativa. Os dados da pesquisa foram obtidos através da análise de prontuários das primeiras consultas de pacientes atendidos no ambulatório de reumatologia das clínicas integradas de uma Universidade do Extremo Sul Catarinense no período de julho de 2016 até julho 2018. Os dados coletados dos prontuários foram: idade, sexo, escolaridade, cidade de procedência, forma de agendamento de consulta, comorbidades prévias à consulta, motivo principal da consulta, diagnósticos ao final do seguimento, medicamentos ao final do seguimento, conduta e diagnósticos ao final em pacientes com perda do seguimento.

Foram considerados perda de seguimento os pacientes que não agendaram retorno ao atendimento ou que não compareceram à consulta previamente agendada, até o prazo final do período do estudo. Com relação aos medicamentos registrados, os modificadores do curso de doença (MMCD) sintéticos incluíram o uso de hidroxicloroquina, metotrexato, leflunomida e sulfassalazina, azatioprina e micofenolato de mofetila. Os MMCD biológicos incluíram o uso de infliximabe, adalimumabe, certolizumabe, golimumabe, etanercepte, rituximabe e secuquinumabe. Suplementos alimentares foram definidos como uso de suplementos de cálcio, suplementos de vitamina $D$, suplementos de colágeno, condroitina, glicosamina, ou associação entre estes suplementos.

Os dados coletados foram organizados e analisados com auxílio do software IBM Statistical Package for the Social Sciences (SPSS) versão 21.0. As variáveis qualitativas foram expressas por meio da frequência e porcentagem. Após verificar distribuição da normalidade pelo teste de Kolmogorov-Smirnov, as variáveis quantitativas foram expressas por meio de média e desvio padrão. As análises inferenciais foram realizadas com um nível de significância $a=0,05$ e, portanto, confiança de $95 \%$. Os resultados foram exibidos por meio de tabelas e gráficos.

O presente estudo foi aprovado pelo Comitê de Ética em Pesquisa e Humanos da Universidade do Extremo Sul Catarinense, sob parecer número 3.034.030. 


\section{RESULTADO}

Foram analisados 218 prontuários referentes às primeiras consultas do ambulatório de reumatologia. Os dados demográficos demonstraram maior prevalência do sexo feminino (83,5\%), com média de idade de 55,9 anos, ensino fundamental incompleto $(59,6 \%)$, sendo $83,9 \%$ dos pacientes procedentes de Criciúma (Tabela 1$)$.

Tabela 1. Características sociodemográficas dos pacientes atendidos no ambulatório de reumatologia no período de julho 2016 até julho de 2018.

\begin{tabular}{ll}
\hline Características Sociodemográficas & $\begin{array}{l}\text { Média } \pm \mathbf{D P}, \mathbf{n}(\%) \\
\mathbf{n}=\mathbf{2 1 8}\end{array}$ \\
\hline Sexo & $182(83,5)$ \\
Feminino & $36(16,5)$ \\
Masculino & $\mathbf{5 5 , 9 2 \pm 1 2 , 5 5}$ \\
Idade (anos) & \\
Fscolaridade & $130(59,6)$ \\
Fundamental incompleto & $30(13,8)$ \\
Médio incompleto & $7(3,2)$ \\
Médio completo & $35(16,0)$ \\
Superior incompleto & $6(2,8)$ \\
Superior completo & $10(4,6)$ \\
Cidade de procedência & \\
Criciúma & $183(83,9)$ \\
Araranguá & $8(3,7)$ \\
Içara & $7(3,2)$ \\
Forquilhinha & $3(1,4)$ \\
\hline
\end{tabular}

Fonte: Dados da Pesquisa, 2019

Observou-se que a grande maioria dos pacientes analisados agendou sua consulta por livre demanda $(82,1 \%)$, de forma espontânea, enquanto somente $17,9 \%$ haviam sido encaminhadas por outro profissional para consulta.

Conforme descritas na Tabela 2, as queixas principais que motivaram o agendamento de consulta foram artralgia/ 
mialgia em $71,1 \%$ dos casos, seguido de dor axial em 18,8\%; 18,3\% para acompanhamento doença reumatológica já diagnosticada; queixas de artrite em $2,3 \%$ dos casos e 1,8\% dos motivos eram em relação à alteração de exames laboratoriais. Vale ressaltar que 14 pacientes apresentaram-se na consulta com mais de uma queixa principal, ponto esse que justifica o número excedente de motivos de consultas (246), ultrapassando o n de 218.

Tabela 2. Motivos da consulta pelos quais os pacientes procuraram o serviço de reumatologia no período de julho 2016 até julho de 2018.

\begin{tabular}{|c|c|}
\hline Motivo principal da consulta & $\begin{array}{l}n(\%) \\
n=218\end{array}$ \\
\hline Artralgia e/ou mialgia & $155(71,1)$ \\
\hline Dor axial & $41(18,8)$ \\
\hline Acompanhamento de doença reumatológica & $40(18,3)$ \\
\hline Artrite & $5(2,3)$ \\
\hline Alteração de exames & $4(1,8)$ \\
\hline Outros & $1(0,5)$ \\
\hline
\end{tabular}

Fonte: Dados da Pesquisa, 2019

Observou-se alta prevalência de comorbidades na amostra, sendo a mais comum Hipertensão arterial Sistêmica (HAS) em 50,9\%, seguido de $22,8 \%$ de pacientes com transtornos psiquiátricos, $16,1 \%$ com diabetes mellitus tipo 2 e 10,2\% obesos. Nos pacientes previamente diagnosticados com doenças reumatológicas autoimunes, a maioria possuía Artrite Reumatoide (52,6\%), seguido por 21,1\% com Lúpus Eritematoso Sistêmico-LES.

Os diagnósticos realizados ao longo do seguimento dos pacientes foram descritos na Tabela 3. O diagnóstico mais comum dos pacientes atendidos foi Osteoartrite em 40,8\% dos casos, seguido por 19,3\% com doenças reumatológicas autoimunes e 14,2\% com doença óssea metabólica. Nos pacientes diagnosticados com doença reumatológica autoimune, 52,4\% desses foram diagnosticados com Artrite Reumatoide, seguido de 23,8\% com LES, 11,9\% com espondiloartropatias, 9,5\% com artrite psoriática e 2,4\% com esclerose sistêmica. É importante ressaltar que 14,7\% dos pacientes atendidos foram diagnosticados com patologias que geralmente são acompanhadas por outras especialidades clínicas ou cirúrgicas, sendo que as mais prevalentes estavam relacionadas a distúrbios musculoesqueléticos de origem traumato-ortopédica $(53,1 \%)$. É importante salientar que 33 pacientes foram diagnosticados com mais de uma enfermidade, obtendo no máximo 3 patologias, justificando novamente o total excedente (261) do valor de n(218). 
Tabela 3. Diagnóstico dos pacientes atendidos na reumatologia no período de julho 2016 até julho de 2018.

\begin{tabular}{ll}
\hline Diagnóstico & $\mathrm{n}(\%)$ \\
& $\mathrm{n}=218$ \\
\hline Osteoartrite & $89(40,8)$ \\
Doença Reumatológica Autoimune & $42(19,3)$ \\
\multicolumn{1}{|c}{ Artrite reumatoide } & $22(52,4)$ \\
LES & $10(23,8)$ \\
Espondiloartropatias & $5(11,9)$ \\
Artrite Psoriásica & $4(9,5)$ \\
Esclerose sistêmica & $1(2,4)$ \\
Doenças relacionadas a outras especialidades & $32(14,7)$ \\
Ortopédicas & $17(53,1)$ \\
Endocrinológicas & $5(15,6)$ \\
Psiquiátricas & $4(12,5)$ \\
Outras & $6(18,8)$ \\
Artrite gotosa & $31(14,2)$ \\
Fibromialgia & $30(13,8)$ \\
Outras doenças reumatológicas & $18(8,2)$ \\
Reumatismo de Partes Moles & $13(6,0)$ \\
\hline & $6(2,8)$ \\
\hline
\end{tabular}

LES: Lúpus Eritematoso Sistêmico

Fonte: Dados da Pesquisa, 2019

Os medicamentos utilizados pelos pacientes ao final do seguimento foram descritos na Tabela 4. 0 uso de anti-inflamatórios não esteroidais (AINEs) representa a classe de medicamento mais utilizada, correspondente a 56,4\%, seguido por suplementos alimentares em $47,2 \%$ da amostra. Estes dados refletem a alta prevalência de osteoartrite neste estudo, visto que estes medicamentos são comumente utilizados no tratamento destas condições. Observou também alta prevalência de uso de antidepressivos (22,0\%). A prevalência do uso de MMCD sintéticos e MMCD biológicos foi baixa, 14,7 e 2,8\% respectivamente, correspondente a prevalência de doenças reumatológicas autoimunes na amostra. Nessa amostragem 80 pacientes fazem uso de duas classes de medicamentos associadas para suas variadas patologias apresentadas, novamente discorrendo a favor de um maior número de medicamentos (378) em relação ao n(218). 
Tabela 4. Medicamentos utilizados pelos pacientes atendidos no ambulatório de reumatologia no período de julho 2016 até julho de 2018.

\begin{tabular}{|c|c|}
\hline Medicamentos utilizados & $\begin{array}{l}n(\%) \\
n=218\end{array}$ \\
\hline Anti-inflamatórios não esteroidais & $123(56,4)$ \\
\hline Suplementos & $103(47,2)$ \\
\hline Antidepressivos & $48(22,0)$ \\
\hline Medicamentos Modificadores do Curso da Doença Sintéticos & $32(14,7)$ \\
\hline Gabapentinoides & $27(12,4)$ \\
\hline Corticoides & $22(10,1)$ \\
\hline Opioides & $17(7,8)$ \\
\hline Medicamentos Modificadores do Curso da Doença Biológicos & $6(2,8)$ \\
\hline
\end{tabular}

Fonte: Dados da Pesquisa, 2019

Dos pacientes analisados, $44,0 \%$ permaneceram em acompanhamento no serviço, $28,5 \%$ receberam alta ambulatorial ou foram encaminhados a outras especialidades e $27,5 \%$ perderam seguimento. Foram consideradas perdas de seguimento pacientes que, em acompanhamento, não remarcaram novas consultas, ou que, remarcaram e não compareceram dentro do prazo estipulado do estudo. Os pacientes com perda de seguimento têm diagnóstico, em maior parte de Osteoartrite (45\%).

\section{DISCUSSÃO}

Os dados demográficos referentes a população estudada foram compatíveis com os observados na literatura, mostrando maior prevalência de doenças reumatológicas em mulheres de meia idade ou mais (50 a 59 anos), responsável por dor crônica como queixa principal, representada principalmente pela presença de osteoartrite. A literatura traz dados mostrando que a cidade de procedência predominante são as capitais devido aos grandes centros de atendimento especializado 7 . Na condição da amostra deste estudo, a cidade de procedência prevalente foi Criciúma, uma vez que é a localização da clínica avaliada.

Observando os meios de agendamento de consultas, verificou-se que a maioria dos pacientes faz agendamento por livre demanda, e apenas em menor porcentagem possui encaminhamento por outros médicos.

Existem evidências consideráveis de que os encaminhamentos podem ser aprimorados para otimizar 0 
atendimento clínico e os resultados dos pacientes, por diagnóstico mais fidedigno e tratamento precoce. Contudo, esse foco falha em considerar a totalidade dos encaminhamentos em que orientações inadequadas podem impactar negativamente a pontualidade das consultas reumatológicas apropriadas, podendo gerar fragmentação do cuidado. No entanto, não há consenso para definir o que constitui um encaminhamento reumatológico adequado ${ }^{10}$.

Além disso, as queixas principais nas consultas relatadas neste estudo, e em estudos prévios, demonstraram que essas queixas estão relacionadas com dor musculoesquelética crônica, como artralgias/mialgias, principalmente periféricas (joelhos e mãos) ${ }^{13}$ e também axial, representado principalmente por lombalgia ${ }^{14}$.

Em relação às comorbidades apresentadas pelos pacientes, a HAS foi a mais prevalente, compatível com a elevada prevalência desta condição na faixa etária apresentada pelos pacientes da amostra ${ }^{15}$. Obesidade apresentou alta prevalência, sendo principalmente um fator de risco importante para desenvolvimento de osteoartrite relacionada à sobrecarga mecânica articular e geração de inflamação sistêmica ${ }^{16}$.

Houve elevada prevalência de transtornos psiquiátricos em nossa amostra. Este dado é compatível com a literatura, que demonstra que até $45 \%$ dos pacientes encaminhados para uma clínica de reumatologia tinham um distúrbio psiquiátrico, e sua presença previa uma menor probabilidade de diagnóstico de uma doença reumatológica autoimune ${ }^{17}$. Adicionalmente, um estudo publicado em 2010 identificou artrite como um fator de risco para o desenvolvimento subsequente de distúrbio psiquiátrico especialmente entre pacientes com menos de 45 anos de idade ${ }^{18}$.

Os diagnósticos finais mais prevalentes após atendimento no ambulatório de reumatologia neste estudo foram compatíveis com a literatura. Observa-se o maior número de osteoartrite, seguido por doenças reumatológicas autoimunes, osteopenia/osteoporose e fibromialgia. Entre as doenças reumatológicas autoimunes, artrite reumatoide aparece em maior número, compatível com os dados da literatura que demonstram ser a doença reumatológica autoimune de maior prevalência e incidência ${ }^{13}$.

Como as queixas do aparelho locomotor podem ser imprecisas, observaram-se diagnósticos relacionados a outras especialidades, principalmente patologias traumato-ortopédicas. A inespecificidade das queixas álgicas apresentadas pelos pacientes e a alta prevalência de ausência de encaminhamento médico para agendamento de consulta descrita em nosso ambulatório pode ter contribuído para este fator.

Em relação aos medicamentos utilizados pelos pacientes no final do seguimento, a prescrição em maior número de AINEs e suplementos alimentares, são compatíveis com a prevalência de diagnóstico de osteoartrite e osteopenia/ osteoporose $^{19}$. A prevalência de uso de MMCD sintéticos de 14,7\%, e MMCD biológicos em 2,8\% dos pacientes analisados é compatível com a prevalência de doenças reumatológicas autoimunes observadas em nossa amostra.

No desfecho dos pacientes avaliados, 44\% seguem em acompanhamento clínico de suas patologias. Doenças reumatológicas geralmente são crônicas, e os pacientes necessitam de consultas frequentes com especialista para 
avaliação de atividade de doença, controle sintomático, renovação de receitas ou laudos médicos. Esses fatores podem ocasionar sobrecarga nos serviços e profissionais envolvidos no cuidado, podendo prejudicar a qualidade do atendimento destes pacientes no longo prazo. Estratégias de encaminhamento de pacientes com doença leve ou em remissão de longa data a serviços de atenção primária podem melhorar estes aspectos, quando bem organizadas ${ }^{5}$.

Observou-se perda de seguimento elevada em nossa amostra, porém suas razões específicas não foram avaliadas. Pode-se inferir, visto prevalência de diagnóstico de osteoartrite nos pacientes com perda de seguimento, que em virtude desta patologia poder ser acompanhada por outras especialidades os pacientes não necessariamente sintam necessidade de acompanhamento com um reumatologista. É importante mencionar, que um estudo demonstrou perda de seguimento de cerca de 30\% em pacientes com doenças reumatológicas autoimunes, mas diferenças de definição de perda de seguimento e diferenças em perfis populacionais dificultam a comparação destes achados com nossos resultados ${ }^{20}$.

Destaca-se, ainda, como limitação do presente estudo, a metodologia de aquisição de dados, realizada através de revisão retrospectiva de prontuários, estando sujeita ao registro desses prontuários.

\section{CONSIDERAÇÕES FINAIS}

Diante dos resultados apresentados conclui-se que o perfil epidemiológico dos pacientes atendidos no serviço de reumatologia do serviço de medicina é compatível com o descrito na literatura, evidenciando a grande prevalência de pacientes do sexo feminino com queixa de dor crônica musculoesquelética.

O principal diagnóstico foi de osteoartrite, seguido de doenças reumatológicas autoimunes, representadas principalmente pela artrite reumatoide. Os medicamentos em uso são compatíveis com os tratamentos propostos para os diagnósticos mais prevalentes. Um número considerável de pacientes permaneceram em seguimento no serviço.

O estudo contribuiu no fornecimento de informações úteis para formulação de estratégias que visem otimizar os serviços prestados e aproveitar melhor seus recursos.

\section{AGRADECIMENTOS}

A Deus, pela graça da vida. Aos familiares e amigos pelo apoio. A Universidade do Extremo Sul Catarinense e seu corpo docente pela dedicação com o aprendizado ao decorrer do curso. Ao Prof. Kristian Madeira pelo auxílio prestado ao longo do trabalho. Nossos agradecimentos também aos funcionários das Clínicas Integradas da UNESC pela contribuição nas etapas do estudo. 


\section{REFERÊNCIAS}

1. Global Burden of Disease Study 2013 Collaborators. Global, regional, and national incidence, prevalence, and years lived with disability for 301 acute and chronic diseases and injuries in 188 countries, 1990-2013: a systematic analysis for the global burden of disease study 2013. Lancet 2015;386(9995):743-800.

2. GBD 2013 Mortality and Causes of Death Collaborators. Global, regional, and national age-sex specific all-cause and cause-specific mortality for 240 causes of death, 1990-2013: a systematic analysis for the Global Burden of Disease Study 2013. Lancet 2015;385(9963):117-171.

3. Donabedian A. The quality of care: how can it be assessed? Jama 1988;260(12):1743-1748.

4. Tong A, Jones J, Speerin R, Filocamo K, Chaitow J, Singh-Grewal D. Consumer perspectives on pediatric rheumatology care and service delivery. J Clin Rheumatol 2013;19(5):234-240.

5. Simon JC, Silva EE, Bredemeier M, Malthchik M, Lima VRB. Avaliação do tempo de espera para consultas de reumatologia em centro de atendimento terciário de Porto Alegre-RS. Revista Amrigs 2008;52(4):303-308.

6. Vieira EWR, Lima TMN, Gazzinelli A. Tempo de espera por consulta médica especializada em um município de pequeno porte de Minas Gerais, Brasil. Reme 2015;19(1):67-71.

7. Da Silva FA, Romagna ES, Da Silva CA. Perfil dos pacientes adultos atendidos em ambulatório de reumatologia em um hospital geral em Porto Alegre - RS. Revista da amrigs 2009;53(3):257-260.

8. Yelin E, Yazdany J, Tonner C, Trupin L, Criswell LA, Katz P, Schmajuk G. Interactions between patients, providers, and health systems and technical quality of care. Arthritis Care Res (Hoboken) 2015;67(3):417-424.

9. Gabriel SE, Wagner JL, Zinsmeister AR, Scott CG, Luthra HS. Is rheumatoid arthritis care more costly when provided by rheumatologists compared with generalists? Arthritis Rheum 2001;44(7):1504-1514.

10. Widdifield J, Tu K, Carter Thorne J, Bombardier C, Michael Paterson J, Liisa Jaakkimainen R, et al. Patter- 
ns of care among patients referred to rheumatologists in Ontario, Canada. Arthritis Care Res (Hoboken) 2017;69(1):104114.

11. Casser HR, Schaible HG. Muskuloskeletaler schmerz. Der Schmerz 2015;29(5):486-495.

12. Harrington JT, Walsh MB. Prelappointment management of new patient referrals in rheumatology: a key strategy for improving health care delivery. Arthritis Rheum 2001;45(3):295-300.

13. Montecucco C, Cavagna L, Caporali R. Pain and rheumatology: An overview of the problem. Eur J Pain Suppl 2009;3(2):105-109.

14. Borenstein D. Mechanical low back pain: a rheumatologist's view. Nat Rev Rheumatol 2013;9(11):643653.

15. Passos VMA, Assis TD, Barreto SM. Hipertensão arterial no Brasil: estimativa de prevalência a partir de estudos de base populacional. Epidemiologia e serviços de saúde 2006;15(1):35-45.

16. Cintra ARS, Aikawa P, Cintra EC. Obesidade versus osteoartrite: muito além da sobrecarga mecânica. Einstein (São Paulo) 2014;12(3):374-379.

17. O'Malley PG, Jackson JL, Kroenke K, Yoon K, Hornstein E, Dennis GJ.. The value of screening for psychiatric disorders in rheumatology referrals. Arch Intern Med 1998;158(21):2357-2362.

18. van 't Land H, Verdurmen J, Ten Have M, Van Dorsselaer S, Beekman A, Graaf R. The association between arthrit is and psychiatric disorders: results from a longitudinal population-based study. J Psychosom Res 2010;68(2):187-193.

19. Barnabe C1, Thanh NX, Ohinmaa A, Homik J, Barr SG, Martin L, et al. Healthcare service utilisation costs are reduced when rheumatoid arthritis patients achieve sustained remission. Ann Rheum Dis 2012;(72):1664-1668.

20. Tien YC, Chiu YM, Liu MP. Frequency of lost to follow-up and associated factors for patients with rheu- 
matic diseases. Plos One 2016;11(3):1-10.

20. Silva ALS, Nascimento ER, Coelho EAC. Nurses practices to promote dignity, participation and empowerment of women in natural childbirth. Esc Anna Nery. [Internet]. 2015 [acesso em 18 jan 2018]; 19(3). Disponível em: http://www.scielo.br/scielo.php?pid=S1414-81452015000300424\&script=sci_arttext\&tlng=en. DOI: http://dx.doi. org/10.5935/1414-8145.20150056.

21. Medeiros ABA, Enders BC, Lira ALBC. The Florence Nightingale's Environmental Theory: A Critical Analysis. Esc Anna Nery. [Internet]. 2015 [acesso em 17 de jan 2018]; 19(3). Disponível em: http://revista.cofen.gov.br/ index.php/enfermagem/article/view/1105. DOI: http://dx.doi.org/10.5935/1414-8145.20150069.

22. Bezerra CMB, Silva BCO, Silva RAR, Martino MMF, Monteiro, Enders BC. Análise descritiva da teoria ambientalista de enfermagem. Enferm. Foco. [Internet]. 2018 [acesso em 05 fev 2019]; 9(2). Disponível em: https://doi. org/10.21675/2357-707X.2018.v9.n2.1105.

23. Bochnia ER, Maneira N, Trigueiro TH, Favero L, Kochla KRA, Oliveira FAM. Performance of obstetric nurses in planned home birth. Cienc Cuid Saude. [Internet]. 2019 [acesso em 29 jul 2019]; 18(2). Disponível em: http:/l periodicos.uem.br/ojs/index.php/CiencCuidSaude/article/view/41570. DOI: http://dx.doi.org/10.4025/cienccuidsaude. v18i2.41570.

24. Silva TC, Bisognin P, Prates LA, Bortoli CFC, Oliveira G, ResselLB. Labor and birth care practices an integrative review. Revista de Enfermagem do Centro-Oeste Mineiro. [Internet]. 2017 [acesso em 28 jan 2018];7(e1294). Disponível: em: http://www.seer.ufsj.edu.br/index.php/recom/article/view/1294. DOl: http://dx.doi.org/10.19175/recom. v7i0.1294. 